\title{
A NEW VERTICAL SEISMOGRAPH
}

\author{
By Hugo BentofF
}

The vertical seismograph herein described makes use of a mechanical system of short period in combination with an electro-mechanical transducer $^{1}$ of high sensitivity coupled to a recording galvanometer. In the Galitzin seismograph the transducer consists of a coil which moves in a magnetic field in such a manner as to generate electromotive force proportional to the velocity of the inertia reactor. The present transducer embodies a modification of the common telephone receiver principle and is also of the velocity type.

The mechanical system consists of a cylindrical inertia reactor of 100 kilograms mass supported directly by a helical spring of such stiffness as to provide a period of 0.5 of a second approximately. The pendulum is constrained to move in a vertical line by six bicycle spokes arranged in such a way as to provide substantially a double diaphragm support. Critical damping is maintained by means of a perforated disk moving in a cylindrical container filled with oil. The container is turned out of the top of the inertia reactor. The amount of damping is controlled by varying the separation of the damping disk from the bottom of the container by means of capstan nuts provided for the purpose. Stops in the form of screws are arranged to limit the motion of the reactor during severe disturbances to about 0.5 of a millimeter.

The transducer is placed below the inertia reactor. The permanent magnet is screwed to an adjusting plate which is fastened to the base of the seismometer. Pole-pieces of Armco iron are fastened vertically to the poles of the magnet. A rectangular bar of Armco iron rigidly attached to the lower face of the reactor forms the working air-gaps with the pole-pieces. The length of the gaps is approximately 0.25 to 2 millimeters depending upon the desired sensitivity. A magnetic shunt, connected across the poles of the permanent magnet, is in the form of a

I An electro-mechanical transducer is a device actuated by power from a mechanical system and supplying power to an electrical system or vice versa. See report of the standardization committee. The Journal of the Acoustical Society of America, 2, 324, January, 1931. 
rectangular bar of Armco iron with an air-gap in the middle for regulating the reluctance. The coils are wound around the pole-pieces. The force due to the constant magnetic flux through the working gaps is added to that of gravity so that the extension of the spring is a few millimeters greater than it would be were gravity acting alone. It will be shown later that for small displacements of the armature from the rest position, the increment of magnetic force is proportional to the displacement so that the effect of the magnetic force on the mechanical system is that of a negative stiffness. This is added to the positive stiffness of the spring, with the result that the period of the pendulum is increased a few tenths of a second over the value it would have without the transducer. If the negative stiffness of the transducer is made comparable in magnitude to the stiffness of the spring, the system becomes unstable. Figures 1, 2, and 3 are photographs of the seismometer taken by Mr. F. C. Henson, in whose shops it was constructed.

\section{Theory of the Electro-Mechanical Transducer}

A schematic representation of the transducer is shown in Figure 4. $M$ is the permanent $U$-magnet. $P, P$ are the pole-pieces around which are wound the coils $C, C . A$ is the armature which forms with the polepieces the working air-gaps of reluctance $R / 2$ each. $S, S$ form the shunt of reluctance $R_{s}$. Movements of the reactor relative to the supporting system of the seismometer vary the length of the working air-gaps and this in turn varies the flux through the gaps. The changes in flux thus induce electromotive forces in the coils and the resulting currents operate the galvanometers. Figure 5 represents the equivalent circuit of the transducer. $F$ is the effective structural magnetomotive force of the permanent magnet in gilberts. $R_{m}$ is the effective reluctance of the permanent magnet in oersteds. $R_{\mathrm{s}}$ is the reluctance of the shunt plus the effective leakage reluctance of the circuit. $R$ is the reluctance of the two working gaps in series. The section of the pole-pieces is taken large enough so that their reluctance is small compared to that of the gaps. In this discussion such effects as hysteresis, eddy currents, and fringing of the lines of force in the vicinity of the air-gaps will be neglected. If $\phi_{m}$ is the effective flux through the permanent magnet, $\phi_{s}$ the flux through the shunt plus the leakage flux, and $\phi$ the flux through the working gaps then

$$
\phi_{m}=\phi_{s}+\phi
$$


Bulletin of the Seismological Society of America Volume 22, Plate 13

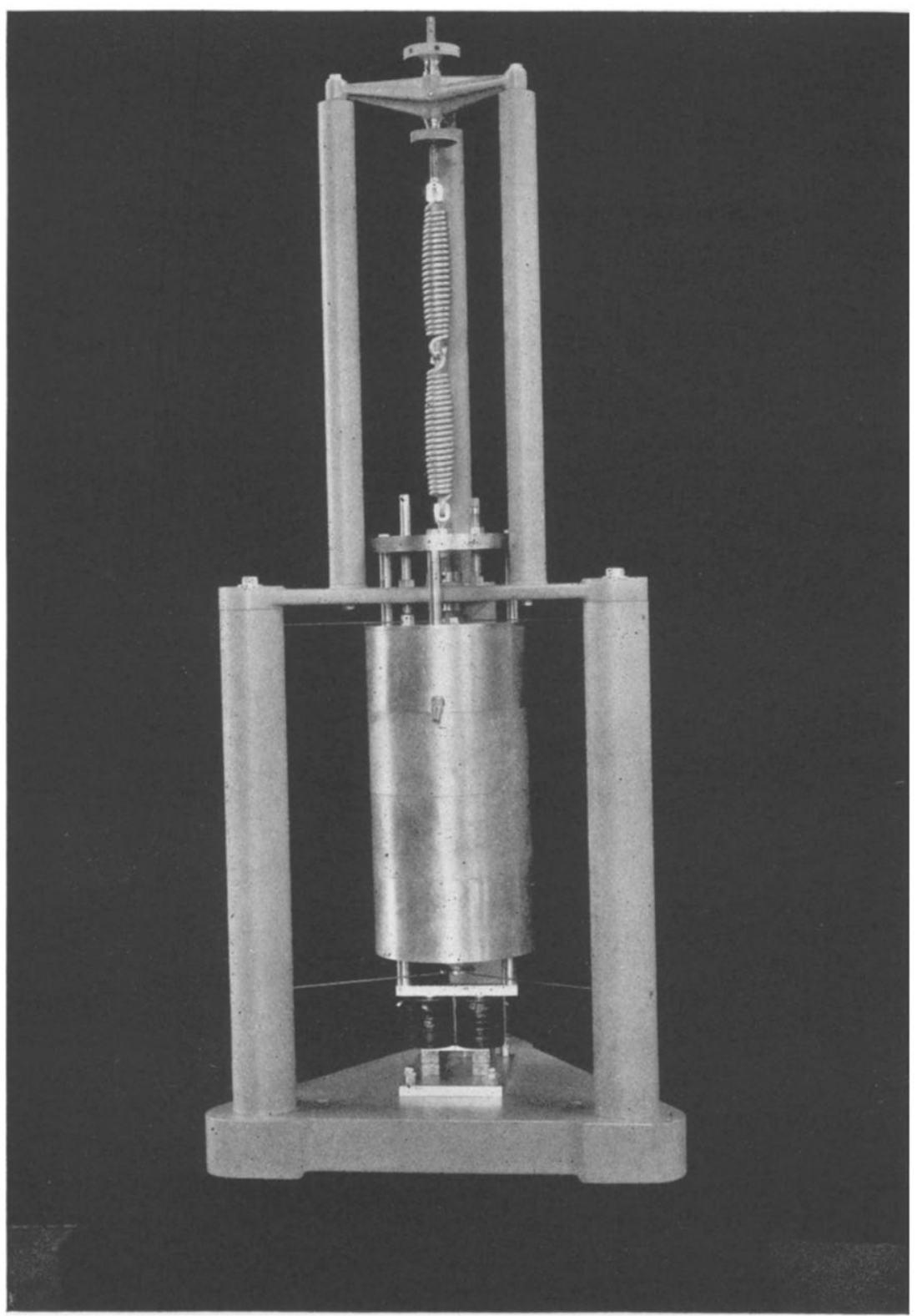

FIG, 1,-Vertical seismometer 
Bulletin of the Seismological Society of America Volume 22, Plate 14

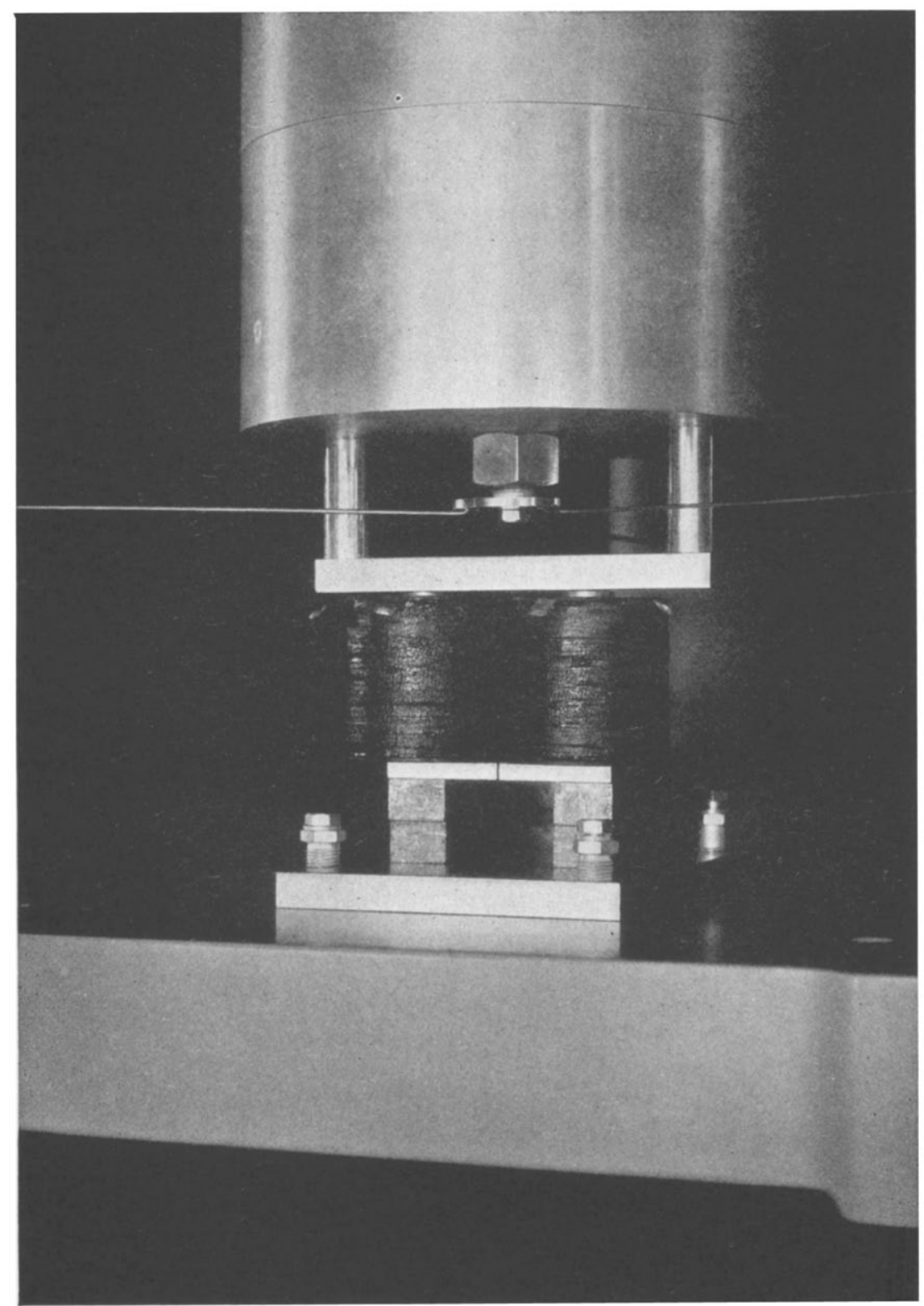

FIG. 2.--Transducer assembly 
Bulletin of the Seismological Society of America Volume 22, Plate 15

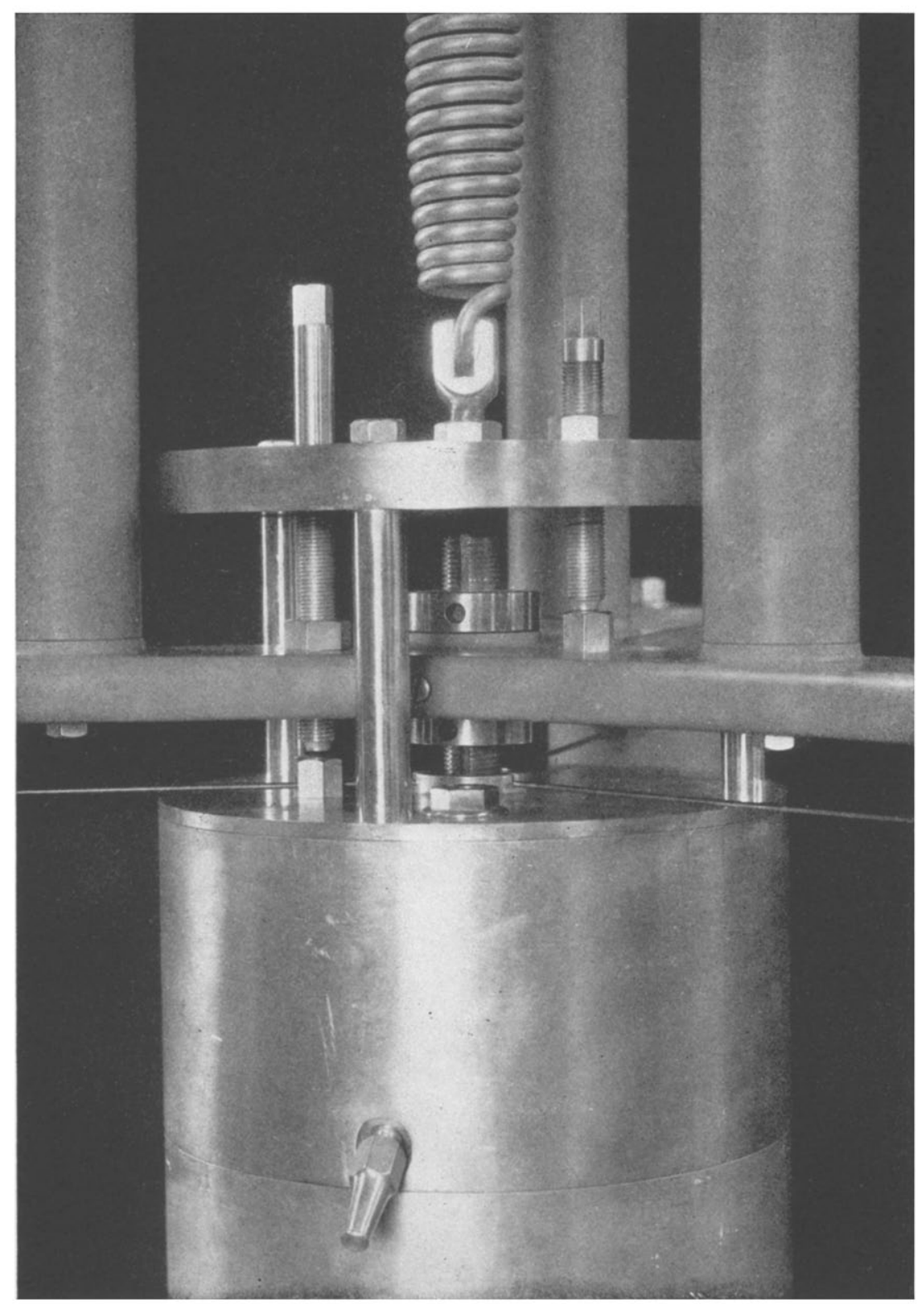

FIG 3-Limit screws and damping adjustment 


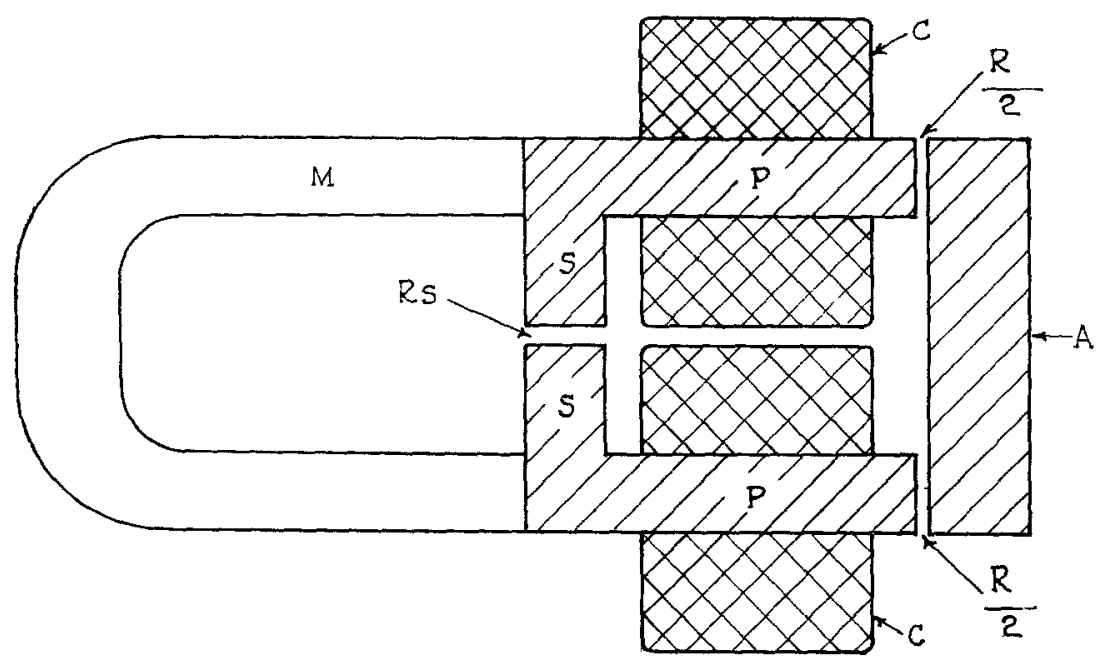

FIG 4.--Schematic representation of transducer

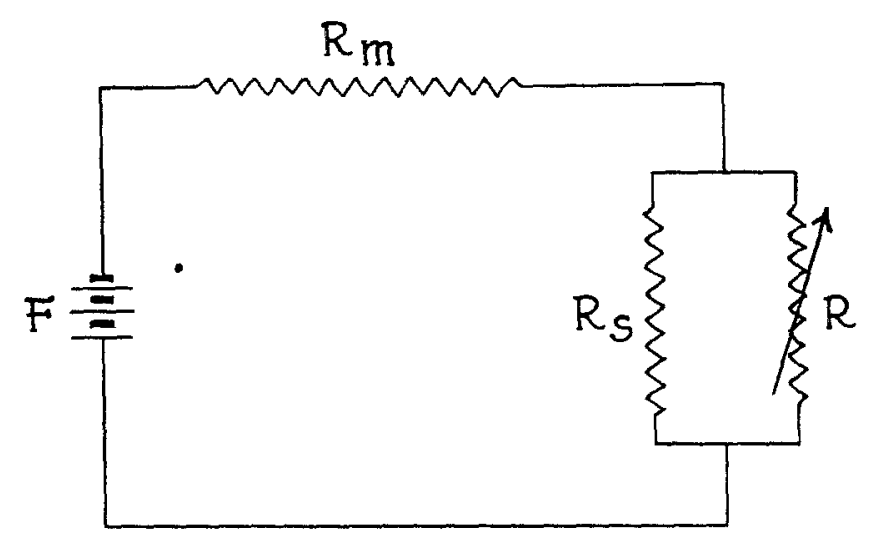

FrG. 5.-Equivalent circut of transducer

and

$$
R_{s} \phi_{s}=R_{\phi}=F-\phi_{m} R_{m}
$$

The solution of Equations 1 and 2 for $\phi$ is

$$
\phi=\frac{F R_{s}}{R R_{m}+R R_{s}+R_{m} R_{s}}
$$


Furthermore

$$
\frac{d \phi}{d R}=-\frac{F\left(R_{s}^{2}+R_{s} R_{m}\right)}{\left(R R_{m}+R R_{s}+R_{m} R_{s}\right)^{2}}
$$

$\frac{d \phi}{d R}$ is a maximum when

$$
R_{s}=\frac{R R_{m}}{R_{m}-R}
$$

If the shunt reluctance of the transducer is adjusted to the value given by Equation 5 then Equation 3 may be written

$$
\phi_{0}=\frac{F}{2 R_{m}} \operatorname{maxwells}
$$

and to a sufficient approximation

$$
\frac{d \phi}{d R}=-\frac{F}{4 R R_{m}}
$$

If $x$ and $s$ are the length and section of each air-gap, then

$$
R=\frac{2 x}{s} \text { oersteds }
$$

and the rate of change of flux through the gaps with respect to the displacement of the armature is

$$
\frac{d \phi}{d x}=\frac{d \phi}{d R} \frac{d R}{d x}
$$

or

$$
\frac{d \phi}{d x}=-\frac{F}{4 x R_{m}}=\frac{\phi_{0}}{2 x_{0}} \text { maxwells per } \mathrm{cm}
$$

In Figure 6 measurements of $\frac{d \phi}{d x}$ are plotted against $\frac{1}{x}$ from three experimental transducers. For correct operation of the seismometer it is necessary that within the range of displacements encountered, $\Delta \phi$ should be linear with respect to $\Delta x$, the armature displacement. In order to de- 


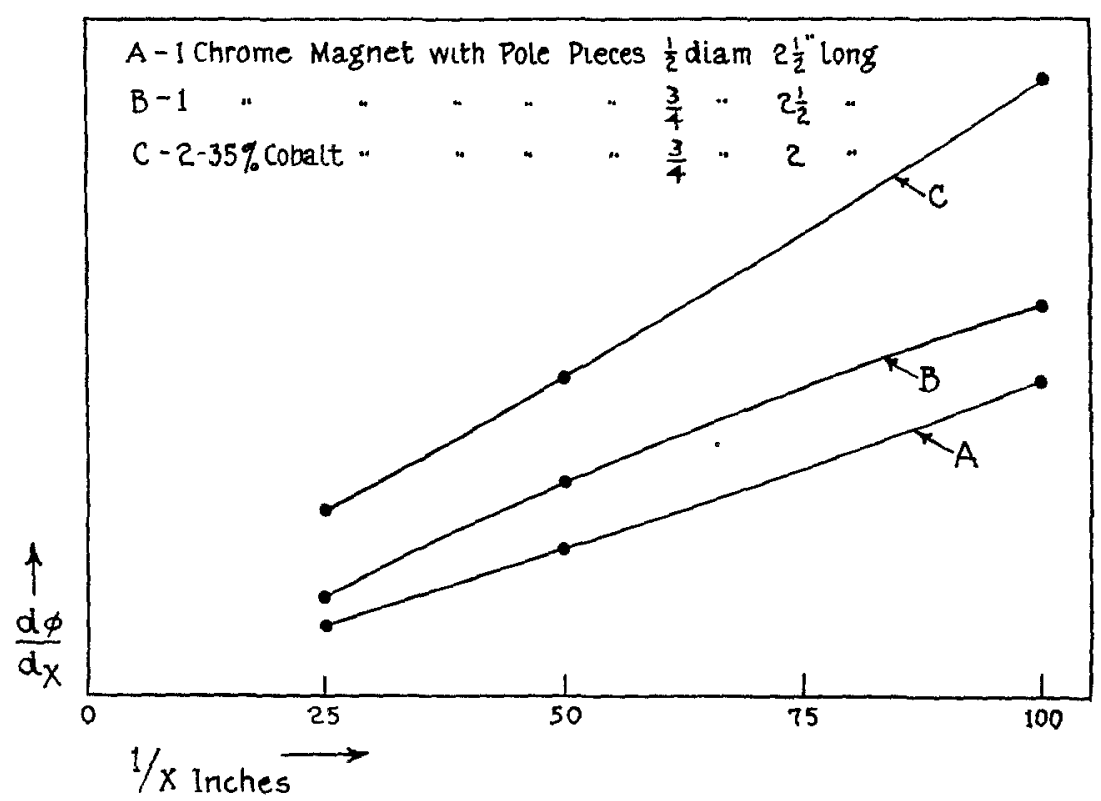

FIG 6.-Relation between sensitivity and length of air-gap

termine how closely this condition is fulfilled we rewrite Equation 3 introducing the value of $R$ from Equation 8

Now let

$$
\phi=\frac{s F R_{s}}{2 x\left(R_{s}+R_{m}\right)+s R_{m} R_{s}}
$$

$$
x=x_{0}(1+\delta) \text { in which } \delta<1
$$

and Equation 10 becomes

in which

$$
\phi_{0}+\Delta \phi=\frac{\phi_{0}}{1+b \delta}
$$

$$
b=\frac{2 x_{0}\left(R_{s}+R_{m}\right)}{2 x_{0}\left(R_{s}+R_{m}\right)+s R_{m} R_{s}}
$$

Since $b$ and $\delta$ are each less than unity, Equation 11 may be written

whence

$$
\phi_{0}+\Delta \phi=\phi_{0}\left[1-b \delta+b^{2} \delta^{2}-b^{3} \delta^{3}+\ldots \ldots\right]
$$

$$
\left.\Delta \phi=-\phi_{0}\left[b \delta-b^{2} \delta^{2}+b^{3} \delta^{3}-\ldots\right]\right]
$$


The departure from linearity in the response is given by the terms in Equation 12, of higher order than the first. Since the signs of the terms alternate, an upper limit for the departure may be computed by taking the second term only. Assuming $b=1$, a value larger than it ever is in practice, the upper limit of the error is therefore $\delta^{2}$, and the ratio of the error to the desired quantity is $\delta$. Since the magnification of the transducer and galvanometer is always greater than $10^{4}$ it follows that for a galvanometer deflection of 10 centimeters the corresponding armature displacement is less than $10^{-3}$ centimeters. If the gap length, $x$, is one millimeter then $\delta$ is $10^{-2}$. The upper limit of the error is thus one per cent.

With a uniform flux density $B$ in an air-gap, the law of magnetic force between the two poles forming the gap is according to Maxwell

$$
f=\frac{B^{2} s}{8 \pi} \text { dynes }
$$

The force on the armature due to the steady flux in the gaps is therefore

$$
f_{0}=-\frac{\phi_{0}^{2}}{4 \pi s} \text { dynes }
$$

The negative sign indicates that the force tends to reduce the gap length $x$.

The behavior of the magnetic force with respect to displacements of the armature from its rest position may be found by introducing values from Equation $11 a$ into Equation 13. Thus if the displacement is $\delta x_{0}$ we may write Equation 13

or

$$
f_{0}+\dot{\Delta} f=-\frac{\phi_{0}^{2}}{4 \pi s}\left[1-b \delta+b^{2} \delta^{2}-b^{3} \delta^{3}+\ldots \ldots\right]^{2}
$$

$$
\left.\Delta f=f_{0}\left[2 b \delta-3 b^{2} \delta^{2}+4 b^{3} \delta^{3}-\ldots\right]\right]
$$

Since terms in $b \delta$ of higher order than the first may be neglected in comparison to $b \delta$ the equation above reduces to

$$
\Delta f=2 f_{0} 6 \delta
$$

which shows that the increment in force is proportional to the displacement rather than to the negative displacement as in the case of springs. The transducer thus adds a negative stiffness of $\frac{2 f_{0} b}{x_{0}}$ dynes per centimeter to the mechanical system and therefore lengthens the period of the pendulum. 
If the number of turns in the coils surrounding the pole-pieces is $n$, the electromotive force induced in the coils as a result of a movement of the armature is

$$
E=n \frac{d \phi}{d t}
$$

Since

$$
\frac{d \phi}{d t}=\frac{d \phi}{d x} \frac{d x}{d t}
$$

we obtain with the help of Equation 9

$$
E=\frac{n \phi_{0}}{2 x_{0}} \frac{d x}{d t} \text { emu }
$$

\section{Theory of the Seismograph}

The equation of motion of the seismometer is

$$
\frac{d^{2} x}{d t^{2}}+2 \varepsilon \frac{d x}{d t}+\omega^{2}{ }_{0} x=-\frac{d^{2} \xi}{d t^{2}}
$$

in which $x=$ the displacement of the inertia reactor relative to the supporting system

$$
\begin{aligned}
\varepsilon & =\text { the damping coefficient } \\
\omega_{0} & =\frac{2 \pi}{T_{0}} \\
T_{0} & =\text { the undamped period of the pendulum } \\
\xi & =\text { the displacement of the earth particles }
\end{aligned}
$$

If

$$
\begin{aligned}
\xi & =a \sin \omega t \\
\omega & =\frac{2 \pi}{T} \\
T & =\text { period of the earth wave }
\end{aligned}
$$

and if the seismometer damping be adjusted to the critical value then Equation 17 becomes

$$
\frac{d^{2} x}{d t^{2}}+2 \omega_{0} \frac{d x}{d t}+\omega_{0}^{2} x=a \omega^{2} \sin \omega t
$$


The steady state solution of Equation 18 is

$$
\begin{aligned}
\frac{d x}{d t} & =\frac{a \omega^{3} \sin (\omega t+\delta)}{\omega^{2}{ }_{0}+\omega^{2}} \\
\delta & =\tan ^{-1} \frac{\omega^{2}{ }_{0}-\omega^{2}}{2 \omega_{0} \omega}
\end{aligned}
$$

Introducing values from Equation 19 into Equation 16 the electromotive force induced in the coils is

$$
E=\frac{n \phi_{0} a \omega^{3} \sin (\omega t+\delta)}{2 x_{0}\left(\omega^{2}{ }_{0}+\omega^{2}\right)}
$$

This electromotive force is applied to the terminals of the galvanometer whose equation of motion ${ }^{2}$ is

$$
\frac{d^{2} \theta}{d t^{2}}+2 \varepsilon_{g} \frac{d \theta}{d t}+\omega^{2} \theta=\frac{g E}{r m}
$$

$\theta=$ angular deflection of galvanometer in radians

$g=$ the electrodynamic constant of the galvanometer, which is the product of the area of the coil, the number of turns, and the field-strength

$r=$ the sum of the galvanometer and transducer resistances

$m=$ the moment of inertia of the galvanometer suspension

$\varepsilon_{g}=$ damping constant of the galvanometer

$\omega_{g}=\frac{2 \pi}{T_{g}}$

$T_{g}=$ the undamped period of the galvanometer

When the mechanical damping of the galvanometer is neglected in comparison to the electromagnetic damping due to reactions of the circuit then

$$
2 \varepsilon_{g}=\frac{g^{2}}{r m}
$$

2 As a result of the large reluctance of the working arr-gaps, the effects of the counter magneto motive force in the transducer are neglected. 
It is evident that in Equation 22 the reactance of the circuit is neglected in comparison to the resistance. Introducing the value of $E$ from Equation 21 the galvanometer equation becomes

$$
\begin{gathered}
\frac{d^{2} \theta}{d t^{2}}+2 \varepsilon_{g} \frac{d \theta}{d t}+\omega_{g}^{2} \theta=\frac{a b \omega^{3} \sin (\omega t+\delta)}{\omega_{0}^{2}+\omega^{2}} \\
b=\frac{g n \phi_{0}}{2 m r x_{0}}
\end{gathered}
$$

The steady state solution of Equation 23 is

$$
\begin{gathered}
\theta=\frac{a b \omega^{3} \sin (\omega t+\delta+\gamma)}{\left(\omega_{0}^{2}+\omega^{2}\right)\left[\left(\omega_{g}^{2}-\omega^{2}\right)^{2}+4 \varepsilon^{2} \omega^{2}\right]^{2}} \\
\gamma=\tan ^{-1} \frac{2 \varepsilon_{g} \omega}{\omega^{2}-\omega^{2} g}
\end{gathered}
$$

Making the following substitutions

$$
\begin{aligned}
& \Delta=\delta+\gamma \\
& Q=\frac{\omega^{3}}{\left(\omega_{0}^{2}+\omega^{2}\right)\left[\left(\omega^{2}{ }_{g}-\omega^{2}\right)^{2}+4 \varepsilon^{2}{ }_{g} \omega^{2}\right]^{\frac{1}{2}}}
\end{aligned}
$$

Equation 24 reduces to

$$
\theta=a b Q \sin (\omega t+\Delta)
$$

If the distance from the galvanometer lens to the recording drum is $L$, then the displacement of the recording light spot on the drum is

$$
D=2 L \theta=2 L a b Q \sin (\omega t+\Delta)
$$

Equations 28 and 29 represent the response of the seismograph for all values of the various constants. ${ }^{3}$ The form of the response characteristic is given by the function $Q . \Delta$ is the phase displacement of the galvanometer relative to the earth displacement.

3 It is clear that Equations 28 and 29 are 1dentical in form with the corresponding equations for the Galitzin seismograph. In spite of this, the new instrument differs markedly in behavior from the normal Galitzin, since the high magnification of the present transducer makes practicable the use of an entirely different combination of seismometer and galvanometer constants. 
An inspection of the function $Q$ shows that a rather wide variation in response characterıstics is available with a single seismometer by merely varying the galvanometer constants. Furthermore the transducer may be arranged with several pairs of coils so that two or more galvanometers may be operated simultaneously by one seismometer. Three galvanometer combinations have been successfully used so far: a Moll micro galvanometer by Kipp \& Zonen with period of 0.20 of a second and critical damping, a Leeds \& Northrup type $\mathrm{R}$ galvanometer with a period of fourteen seconds and critical damping, a galvanometer similar to the preceding but with damping considerably in excess of the critical value. In each case the galvanometer damping is controlled by winding the transducer coils with wire of such size as to provide the required external circuit resistance

The curve $A$ in Figure 7 represents the function $Q$ for the Moll galvanometer combination. The constants used in the computation are $\omega_{0}=12.57$ and $\omega_{g}=31.42$. Since the galvanometer damping is critical $\varepsilon_{g}=\omega_{g}$ and $Q$ reduces to the form

$$
Q=\frac{\omega^{3}}{\left(\omega_{0}^{2}+\omega^{2}\right)\left(\omega_{g}^{2}+\omega^{2}\right)}
$$

This combination is particularly suitable for the recording of local earthquakes since it affords high magnifications for the short-period waves. Furthermore the galvanometer is rugged enough to withstand severe shocks without damage as result of its double suspension and short period. For routine use at this laboratory a magnification in the range of maximum response of approximately 100,000 has given best results. With higher magnifications local traffic microseisms become troublesome. The magnification characteristic corresponding to the routine adjustment is shown in Figure 8, curve A. The high magnifications for periods in the range 0.5 to 2 seconds render this combination very useful in the registration of the first phases of teleseisms.

The curve $C$ in Figure 7 depicts values of $Q$ for the Leeds \& Northrup galvanometer combination with critical damping. In this case also $Q$ is of the form given by Equation 30. The useful magnification of this combination is limited by the microseisms of approximately six seconds period. The magnification characteristic corresponding to the routine adjustment at Pasadena is shown in Figure 8, curve $C$. This represents the highest magnification that can be used during the times of maximum microseismic activity. It can of course be increased considerably when the microseisms are small, but at this laboratory a single adjustment has 


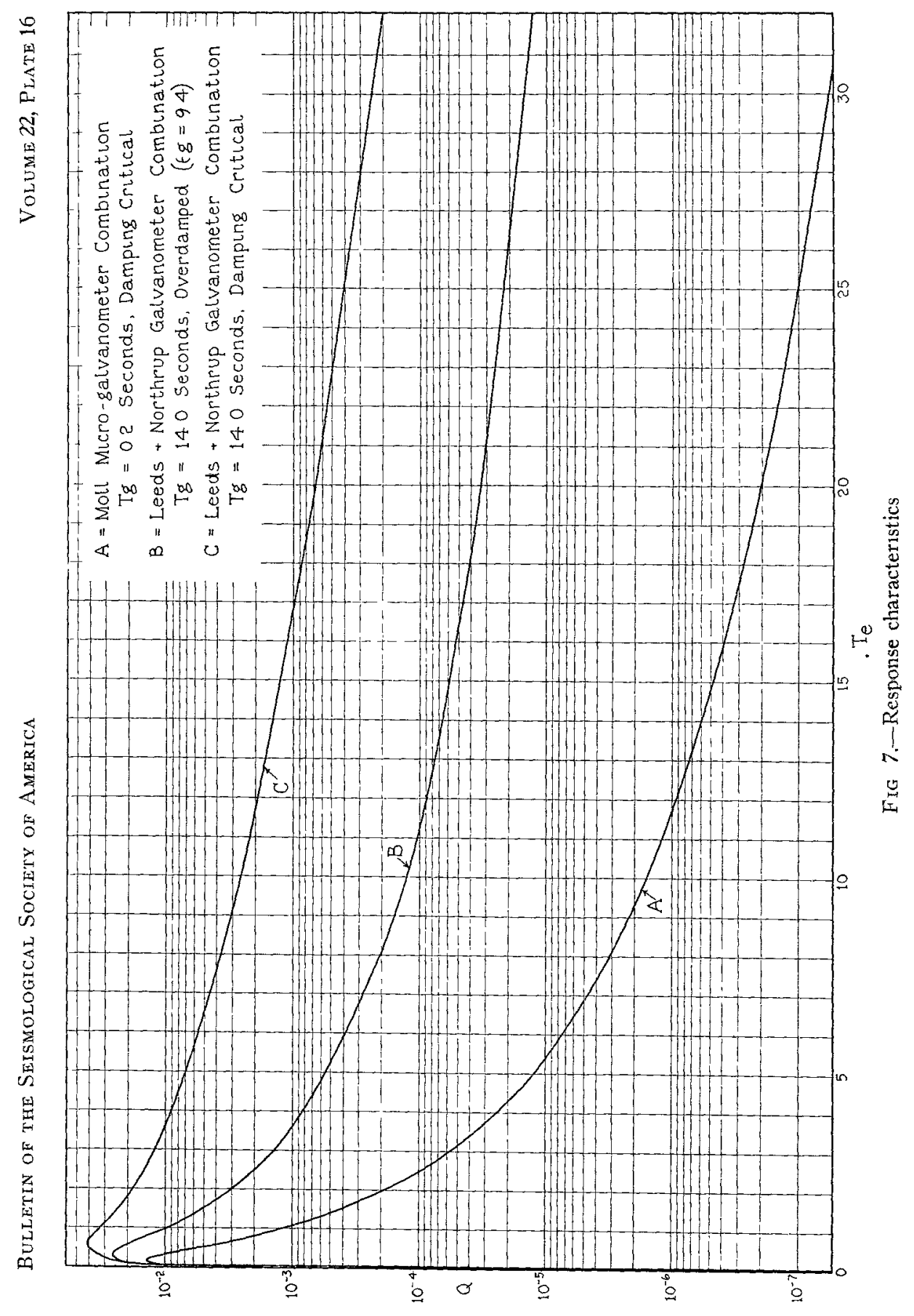




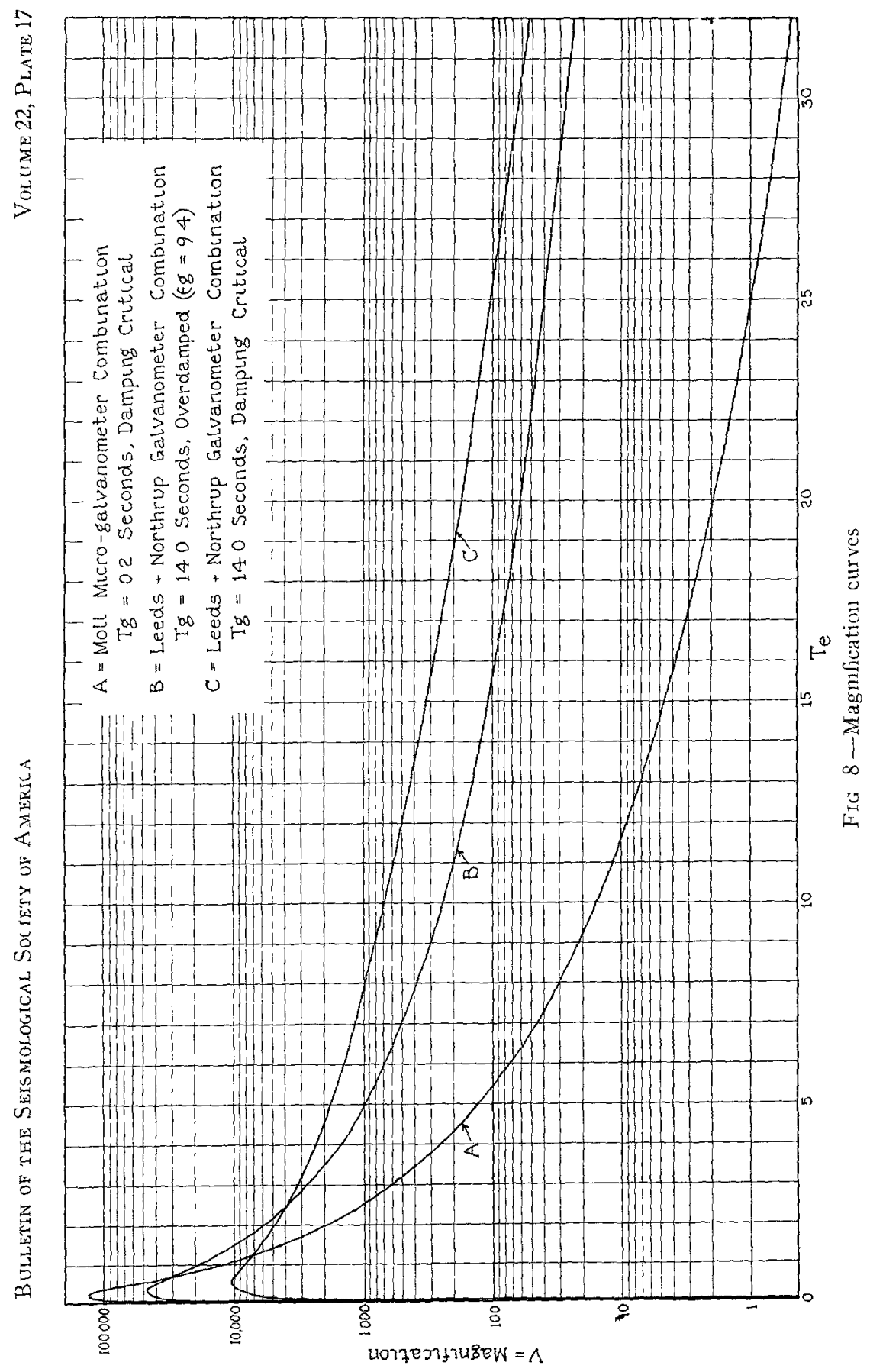


been found satisfactory for all routine work. This is the best combination for the registration of teleseisms. It also records local earthquakes very well. The form of the response curve exhibited by this combination with its relatively high magnification for short periods differs considerably from that of the conventional teleseismic instruments. It is believed, however, that for most routine observations this form is superior, since the waves of various periods in a teleseism are written with approximately equal amplitudes. Thus the important short-period waves of the first phases of the teleseism are clearly recorded, while the later long-period waves are not written so large as to make the reduction of the record difficult. It should be mentioned in this connection that Gutenberg ${ }^{4}$ first constructed an instrument with this type of response characteristic and pointed out its advantages in teleseismic recording. His instrument consisted of a Galitzin seismometer, which he modified by shortening the period of the pendulum and by increasing the magnetic field strength of the transducer. He recorded with a relatively shortperiod galvanometer.

With the Leeds \& Northrup combination in which the damping is considerably greater than the critical value the response characteristic is very nearly the same as that of the mechanical system alone within the useful range of earth periods. The constants for this combination are $\omega_{g}=0.45$ and $\varepsilon_{g}=9.0$. Curve $B$, Figure 7 , represents the function $Q$ for this case. The magnification characteristic is given by $B$, Figure 8 . This combination is suitable for the registration of both local earthquakes and teleseisms, although the magnification for the longer waves is not as great as could be desired. Both this combination and the preceding one are unsuitable for the registration of strong local earthquakes as a result of the fact that severe shocks set up transverse vibrations in the delicate suspensions of the long-period galvanometers.

The magnifications shown in Figure 8 represent the optimum values for routine use at the laboratory in Pasadena. Considerably higher magnifications are avarlable in all three units. When operating singly the magnification of the Moll combination can be increased approximately tenfold and the others about fourfold. With two galvanometers operating simultaneously the increases are about one-half those just given.

In Figure 9 the phase characteristics are given for the three combinations. In computing these values the co-ordinates have been chosen so

4 B. Gutenberg, "Registrierungen mit zwei Galitzinpendeln vershiedener Periode," Beitrage sur Geophysik, 25, 74, 1930. 
that a sudden motion of the earth will produce a corresponding galvanometer response on the records which requires zero phase correction.

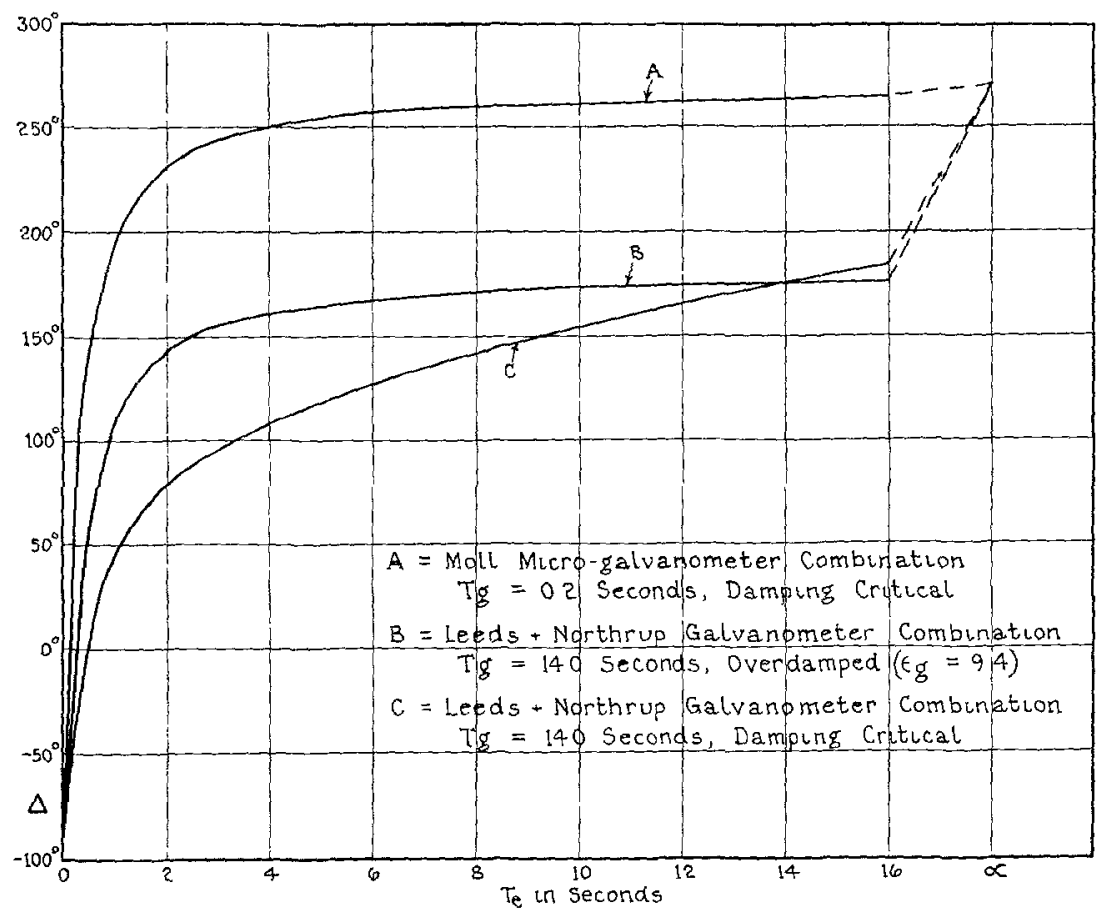

FIG. 9.-Phase characteristics

By winding the transducer conls with very fine wire their impedance can be made high enough to match the input circuit of a vacuum tube amplifier without the use of an input transformer. Connected in this way to an amplifier, the instrument has been used as the pick-up unit for an automatic recording lamp intensifier which increases the brilliance of the recording lights of the routine instruments during the passage of strong earthquakes. A device of this character has been in routine use at th1s laboratory for over a year. Observations made during this interval have clearly demonstrated that the legiblity range of seismograms is greatly extended by its use.

A smaller portable instrument has been constructed. In this model the inertia-reactor has a mass of ten kilograms and a period of approximately 0.3 of a second. With a Moll micro galvanometer the response is 


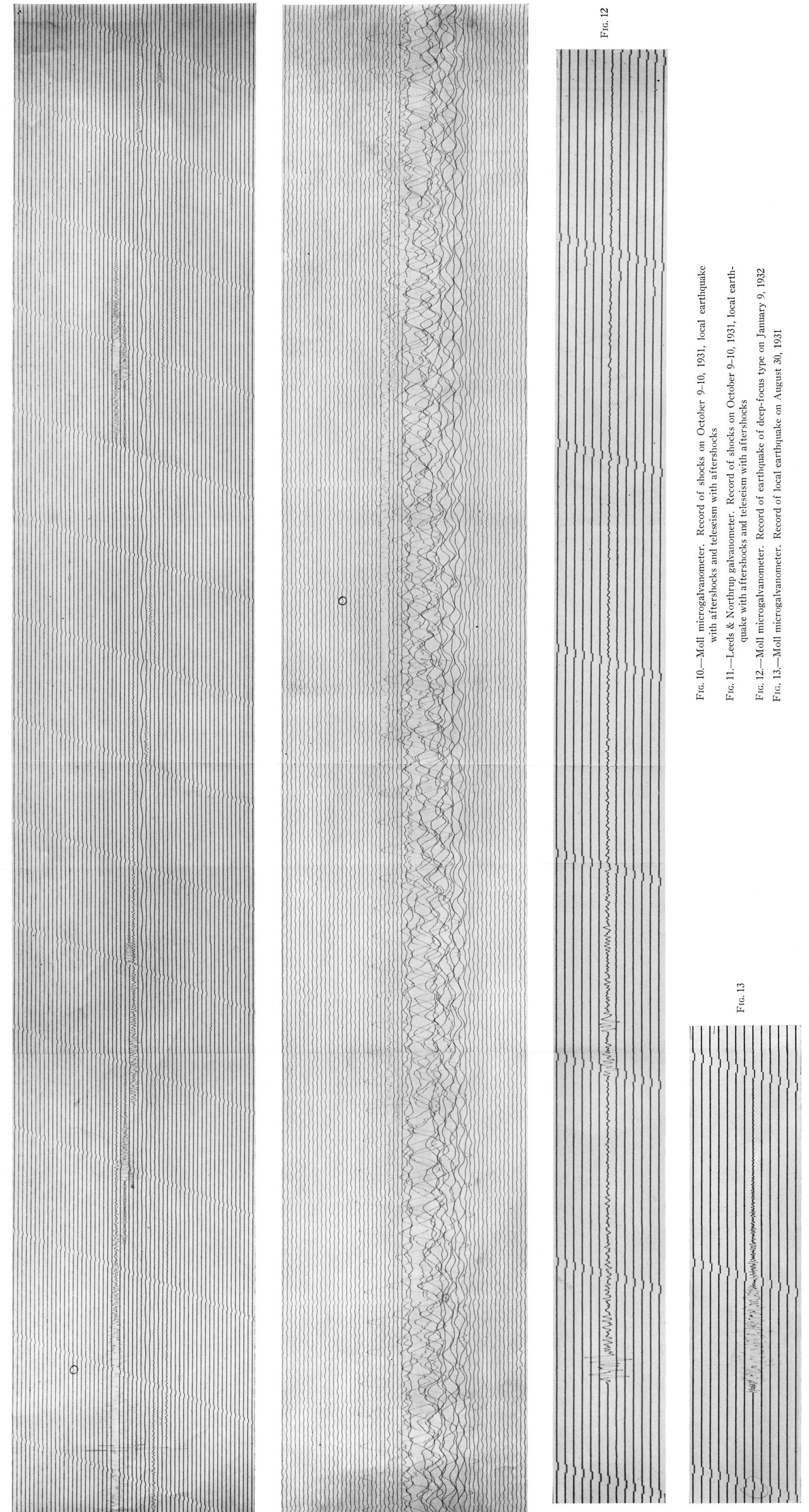


comparable to that of the routine instrument in the registration of local earthquakes. The portable model has also been fitted with high impedance coils and used in connection with a tube amplifier and oscillograph for the registration of blasts.

In Figures 10 and 11 are shown reduced sections of seismograms recorded at Pasadena 1931, October 9-10. The seismogram of Figure 10 was written by the Moll galvanometer combination, and that of Figure 11 was written with the Leeds \& Northrup galvanometer combination adjusted to critical damping. The two galvanometers were operating simultaneously from the same seismometer. On the original records, there are discernible some thirty-two separate shocks originating in three different foc1. The first is a rather strong local earthquake with an origin in the northern part of the Gulf of California. This is followed by a number of aftershocks. The second origin is located some twenty-three kilometers from Pasadena and is represented by a single shock. The third origin is approximately 10,000 kilometers from Pasadena in the vicinity of the Solomon Islands. It 1 s represented by a main teleseism followed by a number of aftershocks. The effectiveness of the Moll combination in exhibiting the first phases of teleseisms 1s clearly seen in Figure 10. The first motion of the main shock is clearly seen on the long-period record in Figure 11, but the aftershocks are difficult to find. As a result of its higher sensitivity for short periods the Moll combination often records the arrivals of the first movements in teleseisms nearly a second earlier than the other instruments at this laboratory. In Figures 10 and 11 the time signal marked with a circle is $21^{\mathrm{h}} 00^{\mathrm{m}} 00^{\mathrm{s}}$ G.C.T , automatically recorded by radio from Annapolis.

Figure 12 shows a record of a deep-focus teleseism which occurred on January 9, 1932. This was written by the Moll combination. It is typical of the appearance of deep-focus earthquakes on the records of this instrument. On the Leeds \& Northrup combination records, this earthquake exhibits somewhat larger surface waves than those usually observed in deep-focus earthquakes.

Figure 13 is a full-scale reproduction of a local earthquake seismogram written with the Moll combination on August 30, 1931. The shock probably occurred on the San Andreas fault, some fifty kilometers due north of Pasadena.

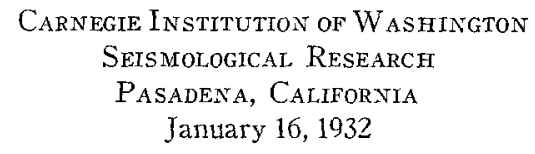

